\title{
Scaling approach to conical indentation in elastic-plastic solids with work hardening
}

\author{
Yang-Tse Cheng a) \\ Physics and Physical Chemistry Department, General Motors Research and Development Center, Warren, \\ Michigan 48090 \\ Che-Min Cheng ${ }^{\text {b) }}$ \\ Laboratory for Non-Linear Mechanics of Continuous Media, Institute of Mechanics, Chinese Academy of \\ Sciences, Beijing 100080, People's Republic of China
}

(Received 24 November 1997; accepted for publication 30 April 1998)

We derive, using dimensional analysis and finite element calculations, several scaling relationships for conical indentation in elastic-plastic solids with work hardening. Using these scaling relationships, we examine the relationships between hardness, contact area, initial unloading slope, and mechanical properties of solids. The scaling relationships also provide new insights into the shape of indentation curves and form the basis for understanding indentation measurements, including nano- and micro-indentation techniques. They may also be helpful as a guide to numerical and finite element calculations of indentation problems. (C) 1998 American Institute of Physics. [S0021-8979(98)04215-7]

\section{INTRODUCTION}

For nearly one hundred years, indentation experiments have been performed to obtain the hardness of materials. ${ }^{1}$ Recent years have seen significant improvements in indentation equipment and a growing need for measuring the mechanical properties of materials on small scales. With the improvement in indentation instruments, it is now possible to monitor, with high precision and accuracy, both the load and displacement of an indenter during indentation experiments in the respective micro-Newton and nanometer range. ${ }^{2-4}$ In addition to hardness, basic mechanical properties of materials, such as Young's modulus, yield strength, and workhardening exponent, may be deduced from the indentation load versus displacement curves for loading and unloading. For example, Oliver and Pharr ${ }^{5}$ and Doerner and $\mathrm{Nix}^{6}$ have proposed methods for determining the hardness and Young's modulus from the peak load and the initial slope of the unloading curves. Finite element methods have also been used to extract the mechanical properties of materials by matching the simulated loading and unloading curves with that of the experimentally determined ones. ${ }^{7-10}$

Recently, significant efforts have been made to better understand the general form of indentation loading and unloading curves. For example, several empirical formulae have been proposed for the loading curves in terms of the Young's modulus and hardness. ${ }^{11,12}$ Loading curves have also been discussed using energetic considerations of reversible and irreversible parts of the indentation induced deformations. ${ }^{13,14}$ Using dimensional analysis and finite element calculations, we have recently derived scaling relationships for indentation in elastic-perfectly plastic solids using conical indenters. ${ }^{15,16}$

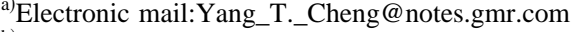

${ }^{b)}$ Electronic mail: zhengzm@LNM.imech.ac.cn
}

In this article, we extend the scaling approach to the analysis of conical indentation in elastic-plastic solids with work hardening. We derive, using dimensional analysis and finite element calculations, simple scaling relationships for loading and unloading curve, initial unloading slope, contact depth, and hardness. We show that hardness is a function of basic mechanical properties of solids, such as Young's modulus, initial yield strength, and work-hardening exponent. The new results are compared with the classic results of Tabor for conical indentation in metals. ${ }^{1,17}$ These scaling relationships provide new insights into the shape of indentation curves and should, therefore, be useful for the interpretation of results obtained by instrumented indentation techniques, including nano- and micro-indentation measurements. They may also be helpful as a guide to numerical and finite element calculations of conical indentation problems.

\section{DIMENSIONAL ANALYSIS}

We consider a three-dimensional, rigid, conical indenter of a given half angle, $\theta$, indenting normally into an elasticplastic solid with work hardening. The stress-strain $(\sigma-\epsilon)$ curves of the solids under uniaxial tension are assumed to be given by

$$
\begin{aligned}
& \sigma=E \epsilon, \text { for } \epsilon \leqslant Y / E, \\
& \sigma=K \epsilon^{n}, \text { for } \epsilon \geqslant Y / E,
\end{aligned}
$$

where $E$ is the Young's modulus, $Y$ is the initial yield stress, $K$ is the strength coefficient, and $n$ is the work-hardening exponent. ${ }^{18}$ To ensure continuity, we note $K=Y(E / Y)^{n}$. Consequently, either $E, Y$, and $K$ or $E, Y$, and $n$ are sufficient to describe the stress-strain relationship. We use the latter set of parameters extensively in the following discussions. When $n$ is zero, Eq. (1) becomes the model for elasticperfectly plastic solids. For most metals, $n$ has a value between 0.1 and $0.5 .{ }^{19}$ We further assume that the friction 


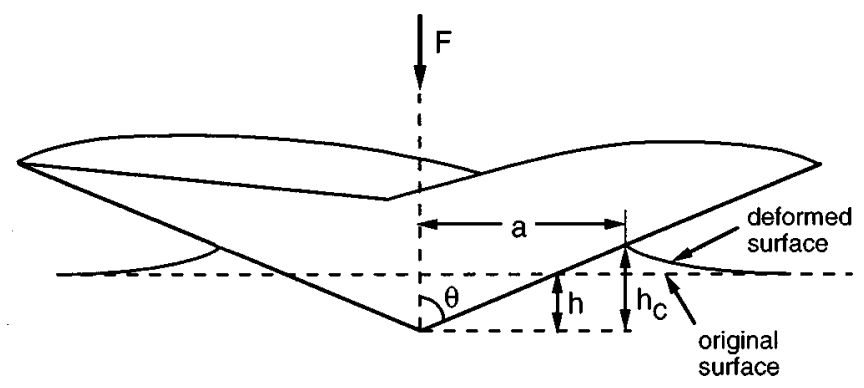

FIG. 1. Illustration of conical indentation.

coefficient at the contact surface between the indenter and the solid is zero. Unlike the cases of indenting into elastic- or rigid-plastic solids, this problem involving elastic-plastic solids with work hardening has no analytical solution.

\section{A. Dimensional analysis of loading}

In general, the quantities of interest from the loading portion of indentation measurements include the force $(F)$ and the contact depth $\left(h_{c}\right)$ (Fig. 1), from which the contact radius $(a)$ and the hardness under load $(H)$ can be evaluated,

$$
a=h_{c} \tan \theta \text {, }
$$

$$
H=\frac{F}{\pi a^{2}} .
$$

For an isotropic elastic-plastic solid obeying the workhardening rule Eq. (1), the two dependent variables, $F$ and $h_{c}$, must be functions, $f_{L}$ and $g$, of all the independent governing parameters, namely, Young's modulus $(E)$, Poisson's ratio $(\nu)$, initial yield strength $(Y)$, work-hardening exponent $(n)$, indenter displacement $(h)$, and indenter half angle $(\theta)$ :

$$
\begin{aligned}
& F=f_{L}(E, \nu, Y, n, h, \theta), \\
& h_{c}=g(E, \nu, Y, n, h, \theta) .
\end{aligned}
$$

Among the six governing parameters, $E, \nu, Y, n, h$, and $\theta$ two of them, namely, $E$ and $h$, have independent dimensions. The dimensions of $Y, \nu, \quad n, \theta, F$, and $h_{c}$ are then given by

$$
\begin{aligned}
& {[Y]=[E],} \\
& {[\nu]=[E]^{0}[h]^{0},} \\
& {[n]=[E]^{0}[h]^{0},} \\
& {[\theta]=[E]^{0}[h]^{0},} \\
& {[F]=[E][h]^{2},} \\
& {\left[h_{c}\right]=[h] .}
\end{aligned}
$$

Applying the $\Pi$ theorem in dimensional analysis, ${ }^{20}$ we obtain:

$$
\begin{aligned}
& \Pi_{\alpha}=\Pi_{\alpha}\left(\Pi_{1}, \nu, n, \theta\right), \text { or equivalently, } \\
& F=E h^{2} \Pi_{\alpha}(Y / E, \nu, n, \theta), \\
& \Pi_{\beta}=\Pi_{\beta}\left(\Pi_{1}, \nu, n, \theta\right), \text { or equivalently, } \\
& h_{c}=h \Pi_{\beta}(Y / E, \nu, n, \theta),
\end{aligned}
$$

where $\Pi_{\alpha}=F / E h^{2}, \Pi_{\beta}=h_{c} / h, \Pi_{1}=Y / E, \nu, n$, and $\theta$ are all dimensionless.

Based on the above dimensional analysis, we can make several observations for a rigid conical indenter with a given half angle, $\theta$, indenting into an elastic-plastic solid with work hardening. First, the force on the indenter, $F$, is proportional to the square of the indenter displacement, $h$. Second, the contact depth, $h_{c}$, is proportional to the indenter displacement, $h$, i.e., the ratio, $h_{c} / h$, is independent of the indenter displacement, $h$, and is a function of $Y / E, \nu, n$, for a given $\theta$. Consequently, the hardness, defined in Eq. (3), is independent of indenter displacement, $h$, or indenter load, $F$.

\section{B. Dimensional analysis of unloading}

Because unloading takes place after loading during which the indenter reaches the maximum depth, $h_{m}$, the force, $F$, is now a function, $f_{U}$, of seven independent governing parameters: Young's modulus $(E)$, Poisson's ratio $(\nu)$, initial yield strength $(Y)$, work-hardening exponent $(n)$, indenter displacement $(h)$, maximum depth $\left(h_{m}\right)$, and indenter half angle $(\theta)$ :

$$
F=f_{U}\left(E, \nu, Y, n, h, h_{m}, \theta\right) .
$$

Dimensional analysis yields

$$
\begin{aligned}
& \Pi_{\gamma}=\Pi_{\gamma}\left(\Pi_{1}, \Pi_{2}, \nu, n, \theta\right), \text { or equivalently, } \\
& F=E h^{2} \Pi_{\gamma}\left(\frac{Y}{E}, \frac{h}{h_{m}}, \nu, n, \theta\right),
\end{aligned}
$$

where $\Pi_{\gamma}=F / E h^{2}, \Pi_{1}=Y / E, \Pi_{2}=h / h_{m}, \nu, n$, and $\theta$ are all dimensionless. In contrast to loading, Eq. (10) shows that the force, $F$, is, in general, no longer simply proportional to the square of the indenter displacement, $h$. It also depends on the ratio, $h / h_{m}$, through the dimensionless function $\Pi_{\gamma}$.

We now consider the initial unloading slope $d F / d h$. Taking the derivative with respect to the indenter displacement and evaluating it at $h_{m}$, Eq. (10) becomes

$$
\left.\frac{d F}{d h}\right|_{h=h_{m}}=E h_{m}\left[\Pi_{\gamma}^{\prime}\left(\frac{Y}{E}, 1, \nu, n, \theta\right)+2 \Pi_{\gamma}\left(\frac{Y}{E}, 1, \nu, n, \theta\right)\right] .
$$

Consequently, the dimensionless quantity,

$$
\begin{aligned}
\left.\frac{1}{E h_{m}} \frac{d F}{d h}\right|_{h=h_{m}} & =\Pi_{\gamma}^{\prime}\left(\frac{Y}{E}, 1, \nu, n, \theta\right)+2 \Pi_{\gamma}\left(\frac{Y}{E}, 1, \nu, n, \theta\right) \\
& \equiv \Pi_{\delta}\left(\frac{Y}{E}, \nu, n, \theta\right),
\end{aligned}
$$

is independent of $h / h_{m}$ and is a function, $\Pi_{\delta}$, of $Y / E, \nu, n$, and $\theta$. Equation (12) shows that the initial unloading slope is proportional to $h_{m}$ in each indentation experiment for which $E, \nu, Y, n$, and $\theta$ are fixed.

\section{FINITE ELEMENT ANALYSIS}

Finite element calculations using $\mathrm{ABAQUS}^{21}$ have been carried out to illustrate the scaling relationships given by Eqs. (7), (8), (12), and to evaluate the dimensionless functions $\Pi_{i}(Y / E, \nu, n, \theta)(i=\alpha, \beta, \delta)$. In a previous study, ${ }^{15,16}$ 
TABLE I. Parameters used in finite element calculations to reveal the scaling relationships in conical indentation using a rigid, conical indenter of half angle $68^{\circ}$ indenting in a solid with a Poisson's ratio 0.3 .

\begin{tabular}{lccccccccccc}
\hline \hline Case & $E(\mathrm{GPa})$ & $Y(\mathrm{GPa})$ & $n$ & Case & $E(\mathrm{GPa})$ & $Y(\mathrm{GPa})$ & $n$ & Case & $E(\mathrm{GPa})$ & $Y(\mathrm{GPa})$ & $n$ \\
\hline 1 & 200 & 0.04 & 0 & 9 & 200 & 2.0 & 0 & 17 & 100 & 9.0 & 0 \\
2 & 200 & 0.08 & 0 & 10 & 200 & 4.0 & 0 & 18 & 100 & 7.0 & 0 \\
3 & 200 & 0.12 & 0 & 11 & 200 & 6.0 & 0 & 19 & 100 & 4.0 & 0 \\
4 & 200 & 0.16 & 0 & 12 & 200 & 8.0 & 0 & 20 & 100 & 1.0 & 0 \\
5 & 200 & 0.4 & 0 & 13 & 200 & 10 & 0 & 21 & 100 & 0.5 & 0 \\
6 & 200 & 0.8 & 0 & 14 & 200 & 12 & 0 & 22 & 100 & 0.1 & 0 \\
7 & 200 & 1.2 & 0 & 15 & 200 & 16 & 0 & 23 & 100 & 0.05 & 0 \\
8 & 200 & 1.6 & 0 & 16 & 200 & 20 & 0 & 23 & 100 & 0.05 & 0 \\
& & & & & & & & & & & \\
24 & 200 & 20 & 0.1 & 39 & 200 & 20 & 0.3 & 54 & 200 & 20 & 0.5 \\
25 & 100 & 8 & 0.1 & 40 & 100 & 8 & 0.3 & 55 & 100 & 8 & 0.5 \\
26 & 200 & 12 & 0.1 & 41 & 200 & 12 & 0.3 & 56 & 200 & 12 & 0.5 \\
27 & 200 & 8 & 0.1 & 42 & 200 & 8 & 0.3 & 57 & 200 & 8 & 0.5 \\
28 & 100 & 2 & 0.1 & 43 & 100 & 2 & 0.3 & 58 & 100 & 2 & 0.5 \\
29 & 200 & 2 & 0.1 & 44 & 200 & 2 & 0.3 & 59 & 200 & 2 & 0.5 \\
30 & 200 & 1.6 & 0.1 & 45 & 200 & 1.6 & 0.3 & 60 & 200 & 1.6 & 0.5 \\
31 & 100 & 0.6 & 0.1 & 46 & 100 & 0.6 & 0.3 & 61 & 100 & 0.6 & 0.5 \\
32 & 200 & 0.8 & 0.1 & 47 & 200 & 0.8 & 0.3 & 62 & 200 & 0.8 & 0.5 \\
33 & 200 & 0.4 & 0.1 & 48 & 200 & 0.4 & 0.3 & 63 & 200 & 0.4 & 0.5 \\
34 & 100 & 0.1 & 0.1 & 49 & 100 & 0.1 & 0.3 & 64 & 100 & 0.1 & 0.5 \\
35 & 200 & 0.16 & 0.1 & 50 & 200 & 0.16 & 0.3 & 65 & 200 & 0.16 & 0.5 \\
36 & 100 & 0.06 & 0.1 & 51 & 100 & 0.06 & 0.3 & 66 & 100 & 0.06 & 0.5 \\
37 & 200 & 0.08 & 0.1 & 52 & 200 & 0.08 & 0.3 & 67 & 200 & 0.08 & 0.5 \\
38 & 200 & 0.04 & 0.1 & 53 & 200 & 0.04 & 0.3 & 68 & 200 & 0.04 & 0.5 \\
\hline \hline
\end{tabular}

we have examined these functions in detail for elasticperfectly plastic solids (i.e., $n=0.0$ ), including the effects of Poisson's ratio $(\nu=0.2,0.3$, and 0.4$)$. For the present calculations, the frequently used half angle of $68^{\circ}$ for the rigid indenter and a typical Poisson's ratio of 0.3 for the solid are chosen to illustrate the essential physics of conical indentation in elastic-plastic solids with work hardening. To simplify notation, $\Pi_{i}(Y / E, n)(i=\alpha, \beta, \delta)$ is used instead of $\Pi_{i}\left(Y / E, 0.3, n, 68^{\circ}\right)(i=\alpha, \beta, \delta)$ in the following discussions.

The rate-independent, incremental theory of plasticity in ABAQUS was used for the finite element calculations. In particular, the plasticity theory uses the Mises yield surface model with associated plastic flow rule. The hardening rule used was that of isotropic hardening and the hardening curves were given by Eq. (1). Table I summarizes the parameters, $E, Y, n$, used for the finite element calculations. The finite element model has been discussed in detail previously. ${ }^{15,16}$ In particular, it was shown that the results of finite element calculations for indentation loading curves approach that predicted by slip-line field theory for rigid-plastic solids and agree with that for elastic solids. ${ }^{15}$

\section{RESULTS AND DISCUSSION}

\section{A. Indentation loading curves}

Figure 2 shows examples of the calculated loading curves for several sets of values of $E, Y$, and $n$. The loading curves were fitted with a power function: $F=a h^{x}$, where $a$ and $x$ are two fitting parameters. The exponent, $x$, obtained from all simulations, such as that shown in Fig. 2 is between 1.98 and 2.03. The finite element calculations thus show that the force, $F$, is proportional to the square of the displacement, $h$, for conical indenter indenting a homogeneous solid with or without work hardening, as expected from dimensional analysis Eq. (7). This square dependency is further illustrated in dimensionless form in Fig. 3 for the loading and unloading curves scaled by the respective maximum depth, $h_{m}$, and maximum force, $F_{m}$. Since work hardening does not change the square dependence, loading curves from conical indentation alone cannot be used to detect whether material work-hardens.

The relationships between $F / E h^{2}$ and $Y / E$ are illustrated in Fig. 4. Clearly, these two quantities lie on a single curve for each value of $n$, as predicted from dimensional analysis. Thus, $F / E h^{2}$ is a function of both $Y / E$ and $n$, i.e., $F$ $=E h^{2} \Pi_{\alpha}(Y / E, n)$. As expected, work hardening has a greater effect on the force required to move the indenter for

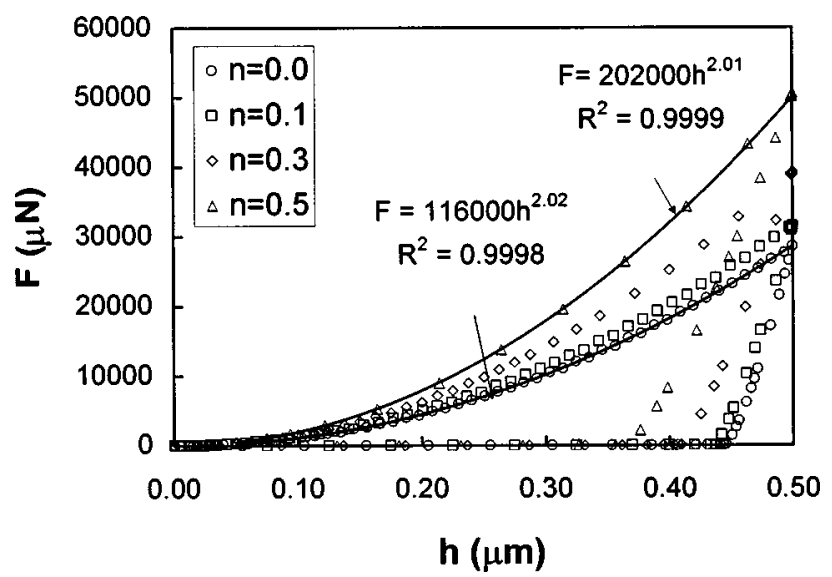

FIG. 2. Examples of indentation curves obtained from finite element analysis: Loading and unloading curves in a solid with $E=200 \mathrm{GPa}, Y=2.0 \mathrm{GPa}$, and several values of $n$. 


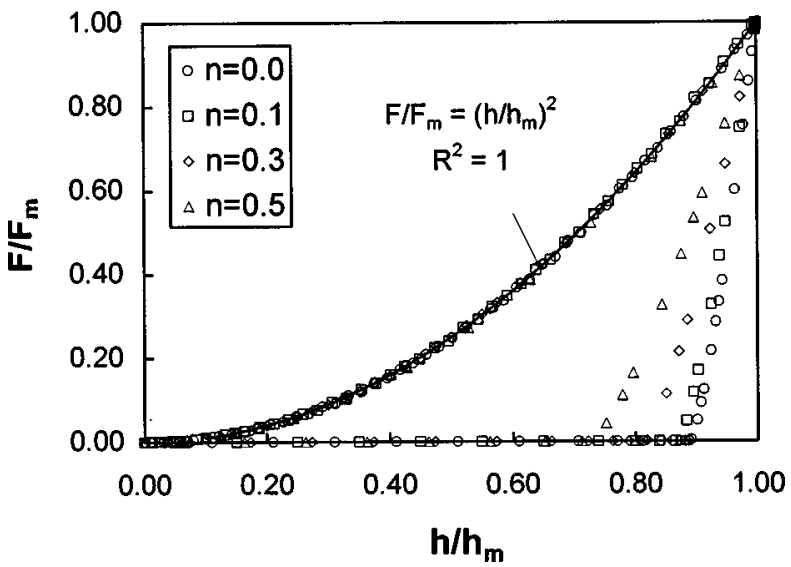

FIG. 3. The same loading and unloading curves shown in Fig. 2 scaled by the respective maximum depth, $h_{m}$, and maximum force, $F_{m}$.

smaller ratio of $Y / E$. For a large ratio of $Y / E$, the value of $F / E h^{2}$ approaches that for purely elastic contact with or without work hardening. Figure 4 shows that, in general, loading curves alone cannot uniquely determine both $Y / E$ and $n$. Thus, caution must be exercised when mechanical properties of solids are extracted by matching the computed indentation loading curves with that obtained from experiments.
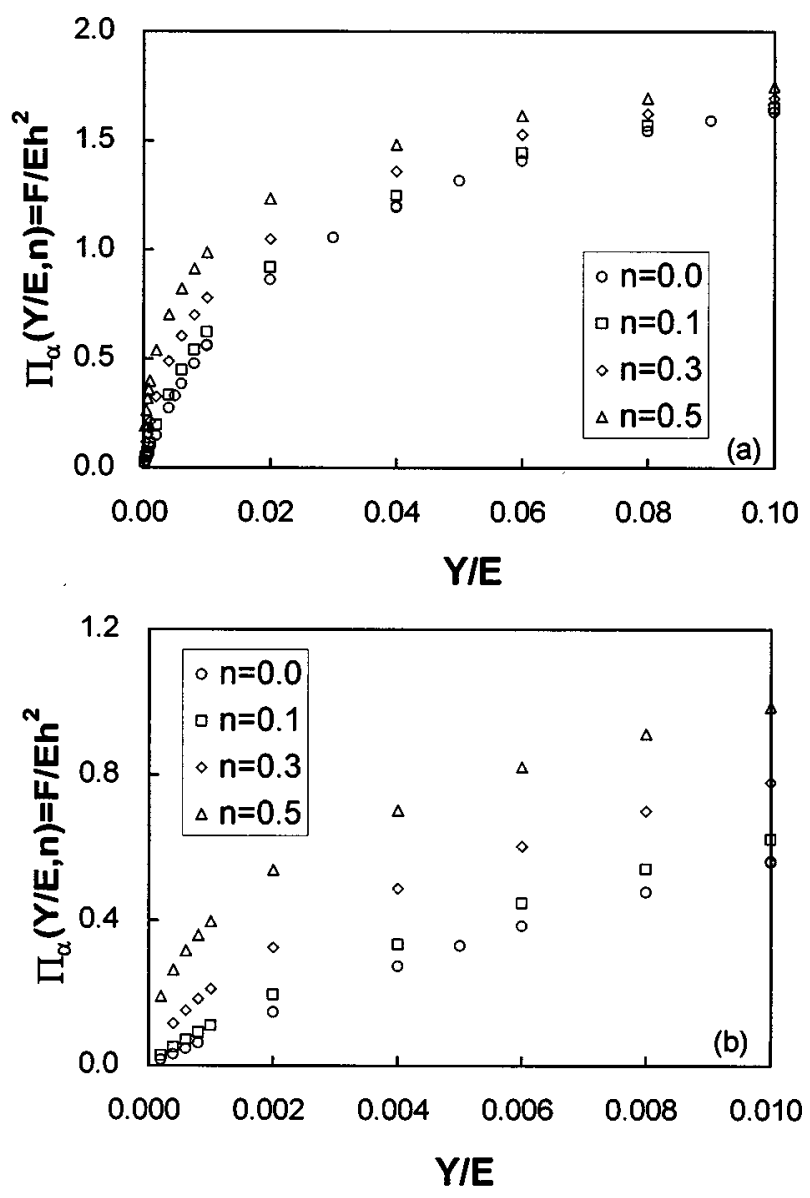

FIG. 4. Scaling relationship for $F / E h^{2}=\Pi_{\alpha}(Y / E, n)$ : (a) For $0.0<Y / E$ $<0.1$; (b) for $0.0<Y / E<0.01$.

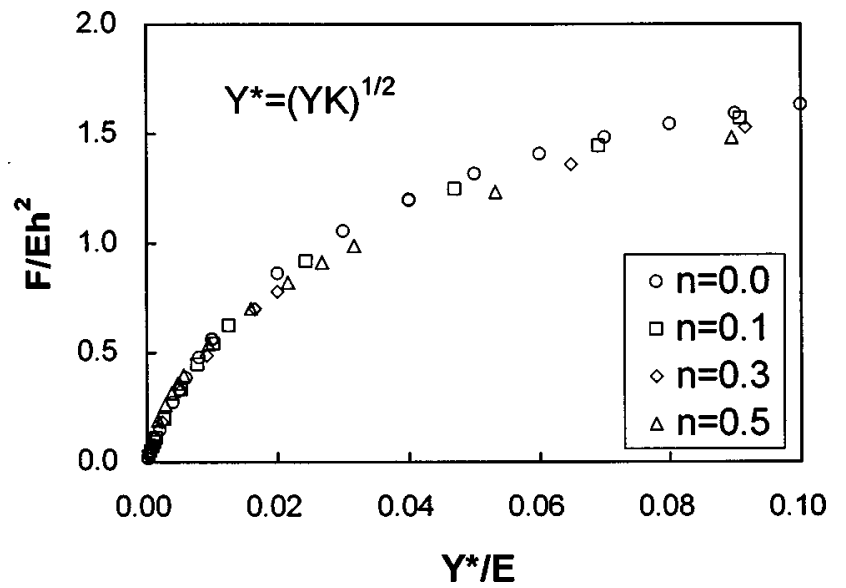

FIG. 5. Approximate scaling relationship between $F / E h^{2}$ and $Y^{*} / E$.

To further study the question of what can be obtained from indentation loading curves, we note that a simple, approximate scaling relationship exists between $F / E h^{2}$ and $Y^{*} / E$, where the "effective yield strength," $Y^{*}$, is given by

$$
Y^{*}=(Y K)^{1 / 2} \text {. }
$$

In Fig. 5, we plot $F / E h^{2}$ vs $Y^{*} / E$. The two quantities lie approximately on a single curve, i.e.,

$$
F=E h^{2} \Pi_{\alpha}(Y / E, n) \approx E h^{2} \Pi_{\alpha}\left(Y^{*} / E, 0\right) .
$$

Consequently, it is possible to estimate the effective yield strength, $Y^{*}$, from indentation loading curves provided that the Young's modulus, $E$, is known. Conversely, $E$ may be obtained if $Y^{*}$ is known.

\section{B. Contact depth, sinking-in, and piling-up}

Figure 6 shows examples of calculated $h_{c}$ vs $h$ for several sets of values of $E, Y$, and $n$. The predicted linear dependence, Eq. (8), is evident. Figure 7 displays the relationship between the calculated $h_{c} / h$ and $Y / E$ for several values of $n$. It is apparent that the ratio $h_{c} / h$ is a function of both $Y / E$ and $n$, as predicted by dimensional analysis. Furthermore, the value of $h_{c} / h$ can be either greater or smaller than

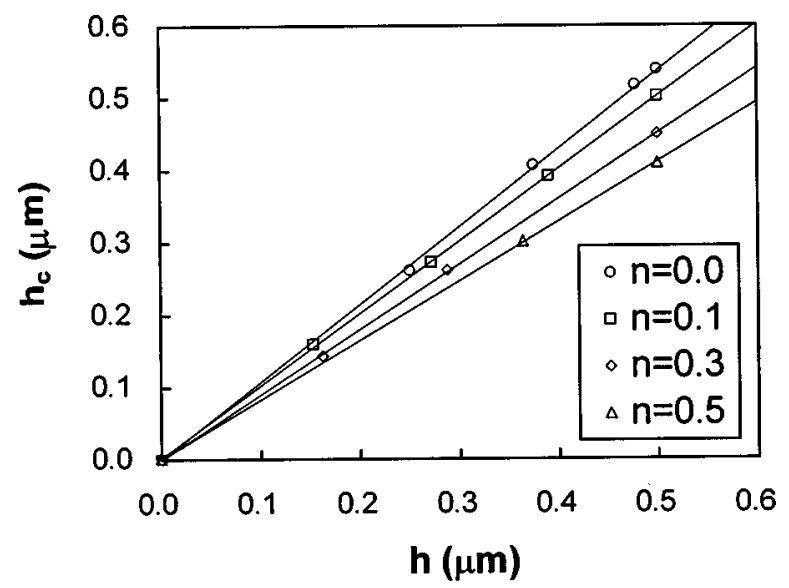

FIG. 6. Examples showing the linear relationship between contact depth, $h_{c}$, and indenter displacement, $h$, obtained from finite element calculations for $E=200 \mathrm{GPa}, Y=2.0 \mathrm{GPa}$, and several values of $n$. 


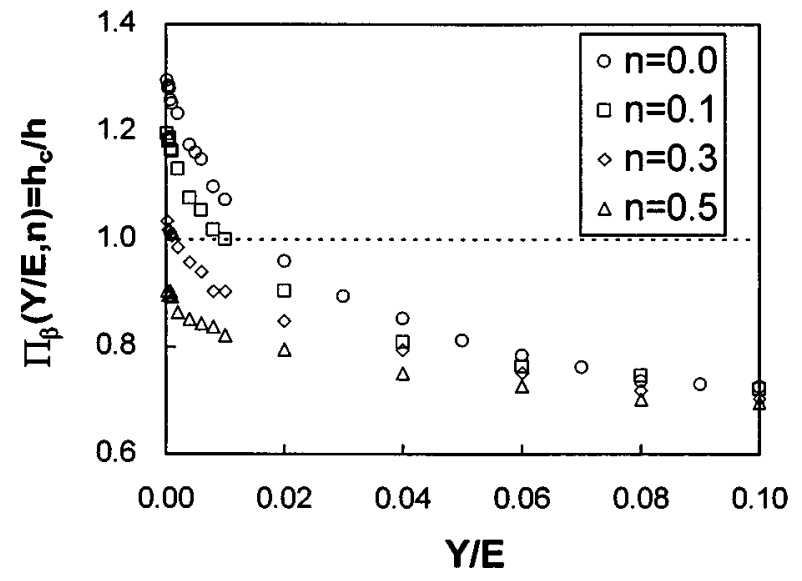

FIG. 7. Scaling relationship for $h_{c} / h=\Pi_{\beta}(Y / E, n)$. The dashed line indicates $h_{c} / h=1.0$.

one, corresponding to the "piling-up" and "sinking-in" of the displaced surface profiles, respectively. For large $Y / E$, sinking-in occurs for all values of $n>0$. For small $Y / E$, both sinking-in and piling-up may occur depending on the degree of work hardening. In the case of severe work hardening (i.e., $n=0.5$ ), sinking-in is expected even for very small values of $Y / E$, whereas piling-up is expected for elasticperfectly plastic solids and for solids with a small workhardening exponent (e.g., $n=0.1$ ).

These simulation results are expected from analytical theories of conical indentation in elastic solids of Sneddon, ${ }^{22}$ where sinking-in occurs, and in rigid-plastic solids of Lockett, ${ }^{23}$ where piling-up occurs. They are also consistent with experimental observations of sinking-in and piling-up phenomena reported in the literature. For example, in metals such as $\mathrm{Cu}$ and mild-steel, where $Y / E$ is small, sinking-in is usually observed in fully-annealed specimens, whereas piling-up is seen in heavily work-hardened samples. ${ }^{17,24,25} \mathrm{In}$ general, therefore, piling-up and sinking-in phenomena are determined by $Y / E$, as well as the work-hardening exponent, $n$.

\section{Initial unloading slope, contact area, and Young's modulus}

Figure 2 also shows examples of calculated unloading curves. The unloading slope was obtained from a linear fit to the first two or three points on the unloading curves. (Care was taken so that there were sufficient number of points close together to represent initial unloading.) The initial unloading slopes are plotted against the maximum depth $h_{m}$ in Fig. 8 for several values of $h_{m}$. A linear relationship between the initial unloading slope and the maximum depth is evident, confirming the predictions of Eq. (12). From Fig. 9 , it is clear that $\left.\left(1 / E h_{m}\right)(d F / d h)\right|_{h=h_{m}}$ is a function of both $Y / E$ and $n$, i.e., $\left.\left(1 / E h_{m}\right)(d F / d h)\right|_{h=h_{m}}=\Pi_{\delta}(Y / E, n)$, as given by dimensional analysis.

As an application of the scaling relationships, we consider the relationship between the initial unloading slope, the contact radius, and Young's modulus of materials. Using Eqs. (2), (8), and (12) we obtain

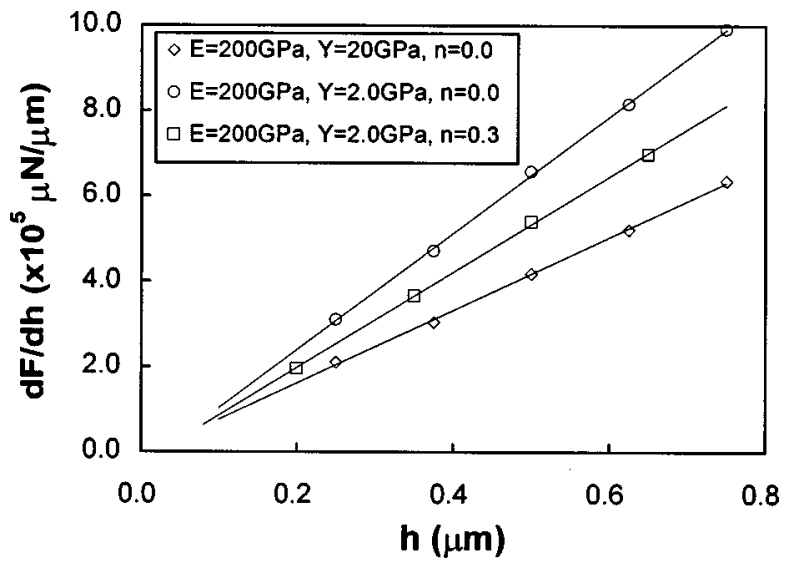

FIG. 8. Examples showing the linear relationship between initial unloading slope, $d F / d h$, and indenter displacement, $h$, obtained from finite element calculations for several values of $E, Y$, and $n$.

$$
\left.\frac{1-\nu^{2}}{E a} \frac{d F}{d h}\right|_{h=h_{m}}=\frac{1-\nu^{2}}{\tan \theta}\left[\frac{\Pi_{\delta}\left(\frac{Y}{E}, \nu, n, \theta\right)}{\Pi_{\beta}\left(\frac{Y}{E}, \nu, n, \theta\right)}\right] .
$$

We evaluate $\left.\left[\left(1-\nu^{2}\right) / E a\right](d F / d h)\right|_{h=h_{m}}$ for $\theta=68^{\circ}$ and $\nu=0.3$. The results are plotted against $Y / E$ in Fig. 10. It is apparent from Fig. 10 that the quantity $\left[\left(1-\nu^{2}\right) /\right.$ $E a]\left.(d F / d h)\right|_{h=h_{m}}$ is roughly independent of $Y / E$ and $n$. The numerical value, obtained from averaging all the cases presented in Fig. 10, is given by

$$
\left.\frac{1-\nu^{2}}{E a} \frac{d F}{d h}\right|_{h=h_{m}} \approx 2.17 \pm 0.05 .
$$

Equation (16) shows that, in principle, the elastic constant, $E /\left(1-\nu^{2}\right)$, may be calculated from the initial slope of unloading curves, provided that the contact radius, $a$, is known.

Equation (16) should be compared with a general relationship between the initial unloading slope $\left[\left.(d F / d h)\right|_{h=h_{m}}\right]$, the contact radius $(a)$, Young's modulus $(E)$, and Poisson's ratio $(\nu)$ :

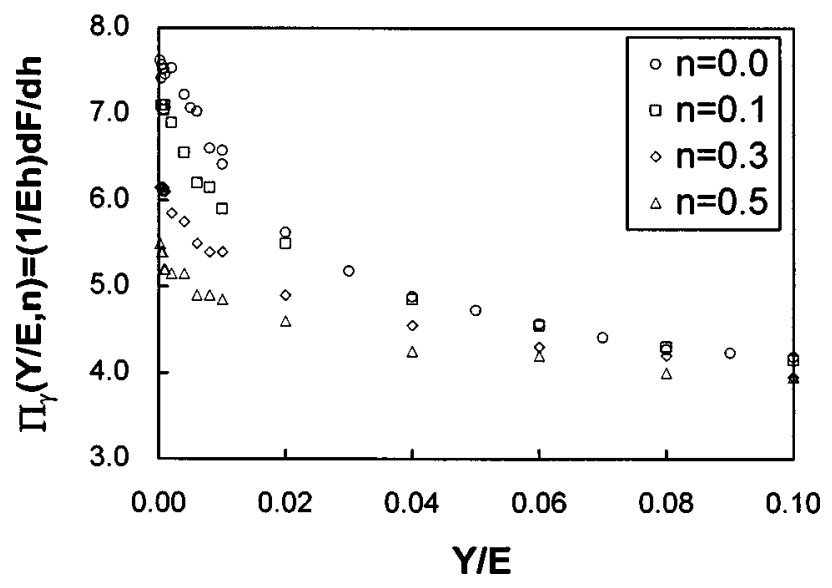

FIG. 9. Scaling relationship for $(1 / E h) d F / d h=\Pi_{\gamma}(Y / E, n)$. 


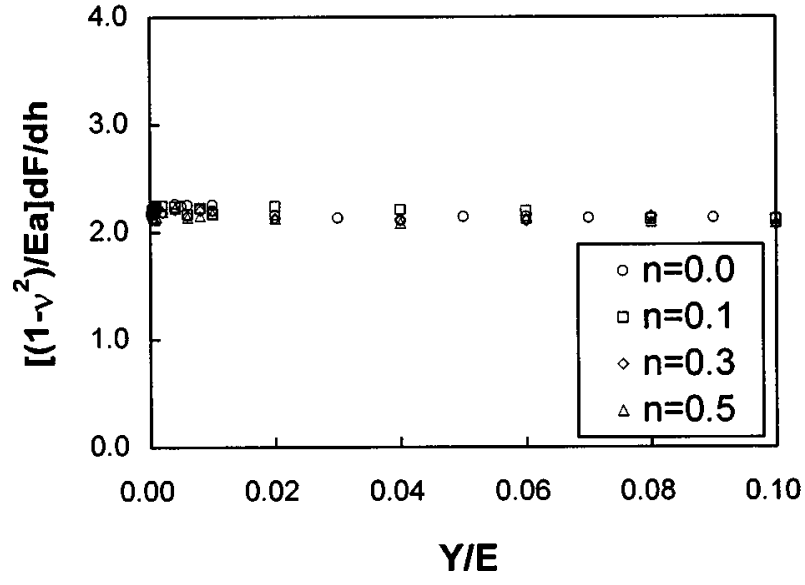

FIG. 10. Relationship between $\left.\left[\left(1-\nu^{2}\right) / E a\right](d F / d h)\right|_{h=h_{m}}$ and $Y / E$.

$$
\left.\frac{1-\nu^{2}}{E a} \frac{d F}{d h}\right|_{h=h_{m}}=2 .
$$

Equation (17) was initially derived for indentation into elastic solids. ${ }^{22,26}$ Using the infinitesimal theory of continuum mechanics, we have recently generalized it to the initial unloading in elastic-plastic solids using indenters with axisymmetric smooth profiles. ${ }^{27}$ Furthermore, we have shown that Eq. (17) holds true even for materials with work hardening and residual stress.

While Eq. (17) was derived using linearized boundary conditions and infinitesimal theory of continuum mechanics, our finite element calculations take into account nonlinear effects, including large strain and moving contact boundaries. Therefore, the small difference in numerical values for $\left.\left[\left(1-\nu^{2}\right) / E a\right](d F / d h)\right|_{h=h_{m}}$ between that given by Eqs. (16) and (17) is not unexpected. In fact, Tanaka and Koguchi $^{28}$ and Bolshakov and Pharr ${ }^{29}$ have shown that [(1 $\left.\left.-\nu^{2}\right) / E a\right]\left.(d F / d h)\right|_{h=h_{m}}$ is about 2.09 and 2.16, respectively, after taking into account the finite radial displacements of points along the surface of contact. While their analyses were for conical indentation in elastic solids, ${ }^{28,29}$ Eq. (16) is a generalization to elastic-plastic solids with work hardening.

\section{Relationship between hardness and mechanical properties}

We now consider the relationship between hardness, elastic and plastic properties of solids. Using Eqs. (2), (3), (7) and (8) the ratio of hardness to initial yield strength is given by

$$
\frac{H}{Y}=\frac{\cot ^{2} \theta}{\pi}\left[\frac{\Pi_{\alpha}(Y / E, \nu, n, \theta)}{(Y / E) \Pi_{\beta}^{2}(Y / E, \nu, n, \theta)}\right] .
$$

Clearly, the hardness is independent of the depth of indentation, $h$. The ratio $H / Y$ is, in principle, a function of $Y / E, \nu$, $n$, as well as indenter geometry $(\theta)$. Taking $\theta=68^{\circ}$ and $\nu=0.3$ for example, the dependence of $H / Y$ on $Y / E$ and $n$ is

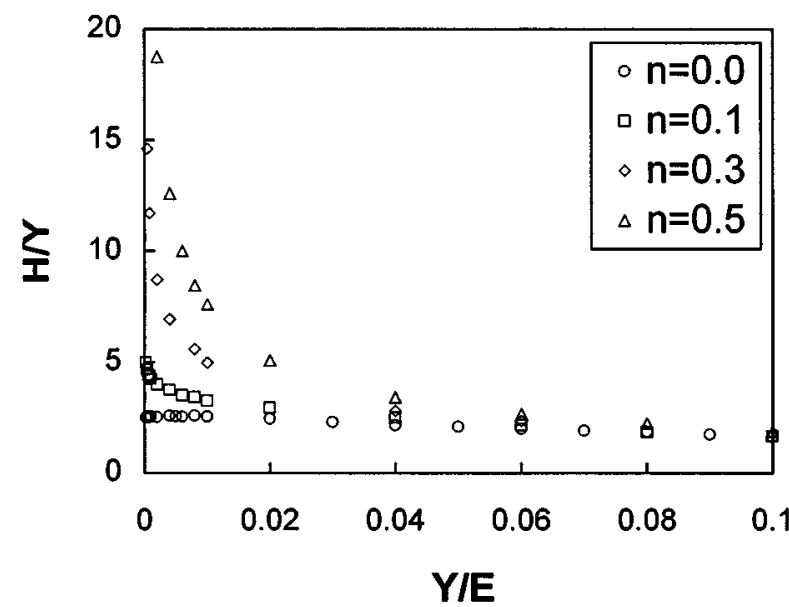

FIG. 11. Relationship between $H / Y$ and $Y / E$ for several values of $n$.

illustrated in Fig. 11. It is apparent that, over the practically relevant range of $Y / E$, the ratio $H / Y$ is not a constant. The hardness, $H$, depends on $Y, E$, and $n$. As expected, work hardening has a greater effect on the hardness value for small ratio of $Y / E$. For large ratio of $Y / E$, the hardness value approach 1.7 times the initial yield strength, $Y$, and is insensitive to $n$. For small ratios of $Y / E$, the hardness value can be many times that of the initial yield strength, $Y$.

Tabor ${ }^{1}$ introduced the concept of "representative yield stress, " $Y_{0}$. He showed that, for conical indentation in metals, the hardness is about 3 times $Y_{0}$, where $Y_{0}$ is the yield stress at a representative strain, $\epsilon_{0}$, of $8-10 \%$. Following Tabor's idea, we evaluated $H /\left(K \epsilon_{0}^{n}\right)$ and noted an approximate scaling relationship between $H /\left(K \epsilon_{0}^{n}\right)$ and $Y / E$, if the value for strain, $\epsilon_{0}$, is taken to be $10 \%$. In Fig. 12, we plot $H /\left(0.10^{n} K\right)$ against $Y / E$. All the data points shown in Fig. 11 lie approximately on a single curve. Thus, the concept of representative strain seems applicable.

It is also evident that $H / Y_{0}$ is a function of $Y / E$ and is, therefore, not a constant over the wide range of $Y / E$ (Fig. 12). For $Y / E<0.02, H / Y_{0}$ is about 2.4 to 2.8 (Fig. 12). For $Y / E>0.06, H / Y$ is about 1.7 to 2.8 (Fig. 11), i.e.,

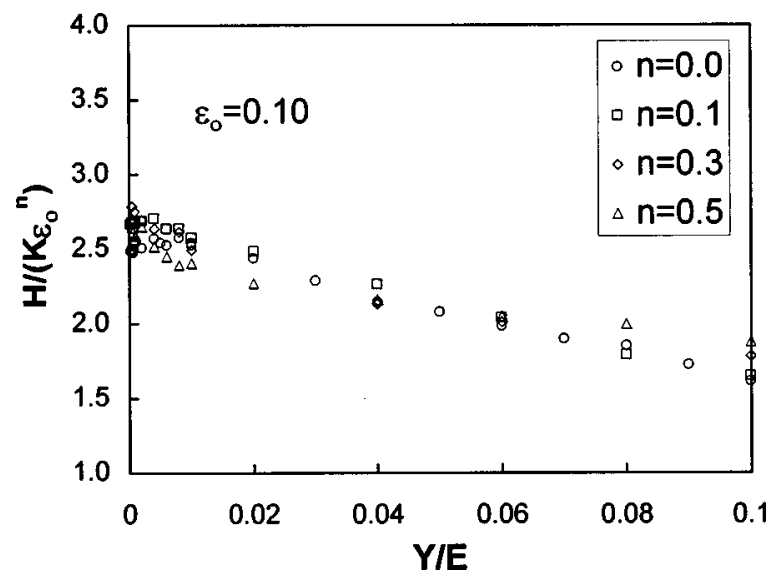

FIG. 12. Relationship between $H /\left(K \epsilon_{0}^{n}\right)$ and $Y / E$ by assuming $\epsilon_{0}=0.10$. 


$$
\begin{aligned}
& H=2.8 Y_{0}, \text { for } Y / E \rightarrow 0.0 \\
& H=1.7 Y, \text { for } Y / E \rightarrow 0.1
\end{aligned}
$$

In practice, it is therefore possible to estimate directly from hardness measurements the representative yield stress, $Y_{0}$, for materials with a small ratio of $Y / E$ and the initial yield stress, $Y$, for materials with a large $Y / E$.

\section{SUMMARY}

Using dimensional analysis and finite element calculations we have derived several scaling relationships for conical indentation in elastic-plastic solids with work hardening. We have shown that, for loading, the force on the indenter is proportional to the square of the indenter displacement. The contact depth is proportional to the indenter displacement. For unloading, the initial unloading slope is proportional to the depth of indentation. These scaling relationships imply:

(1) The "effective"' yield stress, given by the geometric mean of initial yield stress and strength coefficient, i.e., $(Y K)^{1 / 2}$, can be determined from indentation loading curves, provided the Young's modulus is known.

(2) Loading curves alone cannot detect work hardening in materials.

(3) The sinking-in and piling-up of the surface profiles are determined by the ratio of the initial yield stress and Young's modulus, $Y / E$, as well as the degree of workhardening, $n$.

Furthermore, using these scaling relationships we have determined relationships between hardness, contact area, initial unloading slope, and mechanical properties of solids. Specifically, we have shown that, although the contact radius, $a$, and the initial unloading slope, $d F / d h$, at a given indenter displacement are both functions of $Y / E$ and the work-hardening exponent $n$, the ratio, $\left[\left(1-\nu^{2}\right) /\right.$ $E a](d F / d h)$, is roughly a constant. Thus, the elastic constant, $E /\left(1-\nu^{2}\right)$, can indeed be evaluated from the initial unloading slope, provided that the contact radius, $a$, is known.

We have also shown that the ratio of hardness to the initial yield strength $(H / Y)$ is a function of the ratio of initial yield strength to Young's modulus $(Y / E)$ and workhardening exponent $n$. For large $Y / E, H$ is about $1.7 Y$ and is relatively insensitive to $n$. The initial yield strength can, therefore, be estimated from hardness measurement. An approximate scaling relationship between $H /\left(K \epsilon_{0}^{n}\right)$ and $Y / E$ is shown to exist. This implies that Tabor's concept of representative yield stress, $Y_{0}$, which corresponds to a representative strain of $8-10 \%$, applies to conical indentation. However, $H /\left(K \epsilon_{0}^{n}\right)$ is shown to be a function of $Y / E$. This function approaches Tabor's relationship, $H=2.8 Y_{0}$, as $Y / E$ approaches zero.

Although the scaling relationships have been derived for conical indentation, the same approach can be applied to indentation using pyramidal indenters, since pyramidal indenters are also geometrically self-similar. The scaling approach is also not limited by the assumptions of rigid indenter and zero friction between indenter and solid. For example, we consider indentation in elastic-plastic solids with work hardening using an elastic, pyramidal indenter. Let $\Theta$ be the set of angles that describe the shape of a pyramidal indenter, $E_{i}$ the Young's modulus of the indenter, $\nu_{i}$ its Poisson's ratio, and $\mu$ the friction coefficient for the interface between the indenter and solid, dimensional analysis shows that for loading the force is given by

$$
F=E h^{2} \Pi_{\alpha}\left(\frac{Y}{E}, \nu, n, \frac{E_{i}}{E}, \nu_{i}, \mu, \Theta\right),
$$

and the contact area, $A_{c}$,

$$
A_{c}=h^{2} \Pi_{\beta}\left(\frac{Y}{E}, \nu, n, \frac{E_{i}}{E}, \nu_{i}, \mu, \Theta\right) .
$$

Obviously, three-dimensional finite element calculations are needed to evaluate the dimensionless functions in Eqs. (20) and (21). Such calculations, though computationally intensive, are within the current capabilities of commercial finite element software. It is evident, however, that the functional dependence, such as $F \propto h^{2}$ and the independence of hardness on $h$, remains the same as that for conical indentations. We therefore believe that the results for rigid conical indentation presented here capture the essential features of indentation in elastic-plastic solids with work hardening. In fact, the scaling relationships have been used to establish a new correlation between hardness, elastic modulus, and the work of indentation. $^{30}$

\section{ACKNOWLEDGMENTS}

The authors would like to thank W. J. Meng, S. J. Harris, G. L. Eesley, J. R. Smith, L. C. Lev, B. Qiu, S. F. Yin, D. D. Snyder, and K. C. Taylor for helpful discussions. They would also like to thank R. J. Blint, M. S. Meyer, K. Ueno, J. Brown (EDS), P. Lalor (HKS), and L. Hill (HKS) for maintaining the workstations and for helping with the use of ABAQUS. Correspondences with Professor D. Tabor and Professor G. M. Pharr have also been valuable.

${ }^{1}$ D. Tabor, The Hardness of Metals (Oxford, London, 1951).

${ }^{2}$ J. B. Pethica, R. Hutchings, and W. C. Oliver, Philos. Mag. A 48, 593 (1983).

${ }^{3}$ D. Stone, W. R. LaFontaine, P. Alexopoulos, T. W. Wu, and C.-Y. Li, J. Mater. Res. 3, 141 (1988).

${ }^{4}$ B. Bhushan, A. Kulkarni, W. Bonin, and J. Wyrobek, Philos. Mag. A 74, 1117 (1996).

${ }^{5}$ W. C. Oliver and G. M. Pharr, J. Mater. Res. 7, 1564 (1992).

${ }^{6}$ M. F. Doerner and W. D. Nix, J. Mater. Res. 1, 601 (1986).

${ }^{7}$ A. K. Bhattacharya and W. D. Nix, Int. J. Solids Struct. 24, 881 (1988).

${ }^{8}$ T. A. Laursen and J. C. Simo, J. Mater. Res. 7, 618 (1992).

${ }^{9}$ A. Bolshakov, W. C. Oliver, and G. M. Pharr, J. Mater. Res. 11, 760 (1996)

${ }^{10}$ S. M. Myers, J. A. Knapp, D. M. Follstaedt, and M. T. Dugger, J. Appl. Phys. 83, 1256 (1998)

${ }^{11}$ J. L. Loubet, J. M. Georges, and J. Meille, in Microindentation Techniques in Materials Science and Engineering, edited by P. J. Blau and B. R. Lawn (American Society for Testing and Materials, Philadelphia, 1986) p. 72.

${ }^{12}$ S. V. Hainsworth, H. W. Chandler, and T. F. Page, J. Mater. Res. 11, 1987 (1996).

${ }^{13}$ B. Rother, Surf. Coat. Technol. 86-87, 535 (1996).

${ }^{14}$ M. Sakai, Acta Metall. Mater. 41, 1751 (1993).

${ }^{15}$ Y.-T. Cheng and C.-M. Cheng, Philos. Mag. Lett. 77, 39 (1998).

${ }^{16}$ Y.-T. Cheng and C.-M. Cheng, "Scaling laws in conical indentation of 
elastic-perfectly plastic solids," GM Research and Development Center Publication R\&D-8689 (June 23, 1997); Int. J. Solids Struct. (in press).

${ }^{17}$ D. Tabor, Philos. Mag. A 74, 1207 (1996).

${ }^{18}$ J. Lubliner, Plasticity Theory (Macmillan, New York, 1990).

${ }^{19}$ G. Dieter, Mechanical Metallurgy, 2nd ed. (McGraw-Hill, New York, 1976).

${ }^{20}$ G. I. Barenblatt, Scaling, Self-similarity, and Intermediate Asymptotics (Cambridge University Press, Cambridge, 1996).

${ }^{21}$ ABAQUS, version 5.6, Hibbitt, Karlsson \& Sorensen, Inc. Pawtucket, RI 02860.

${ }^{22}$ I. N. Sneddon, Int. J. Eng. Sci. 3, 47 (1963).

${ }^{23}$ F. J. Lockett, J. Mech. Phys. Solids 11, 345 (1963).
${ }^{24}$ M. M. Chaudhri and M. Winter, J. Phys. D Appl. Phys. 21, 370 (1988).

${ }^{25} \mathrm{~S}$. Bec, A. Tonck, J.-M. Georges, E. Georges, and J.-L. Loubet, Philos. Mag. A 74, 1061 (1996).

${ }^{26}$ G. M. Pharr, W. C. Oliver, and F. R. Brotzen, J. Mater. Res. 7, 613 (1992).

${ }^{27}$ C.-M. Cheng and Y.-T. Cheng, Appl. Phys. Lett. 71, 2623 (1997).

${ }^{28}$ K. Tanaka and H. Koguchi, Jpn. J. Tribol. 40, 129 (1995).

${ }^{29}$ A. Bolshakov and G. M. Pharr, Mater. Res. Soc. Symp. Proc. 436, 189 (1997).

${ }^{30}$ Y.-T. Cheng and C.-M. Cheng, "Relationships between hardness, elastic modulus, and the work of indentation," GM Research and Development Center Publication R\&D-8766 (February 6, 1998). 